University of Wollongong

Research Online

Faculty of Engineering - Papers (Archive)

Faculty of Engineering and Information

Sciences

$1-1-2010$

\title{
Vertical absorption edge and temperature dependent resistivity in semihydrogenated graphene
}

Lei Chen

Peking University

Zhongshui Ma

Peking University, Beijing, China, zma@uow.edu.au

Chao Zhang

University of Wollongong, czhang@uow.edu.au

Follow this and additional works at: https://ro.uow.edu.au/engpapers

Part of the Engineering Commons

https://ro.uow.edu.au/engpapers/4032

\section{Recommended Citation}

Chen, Lei; Ma, Zhongshui; and Zhang, Chao: Vertical absorption edge and temperature dependent resistivity in semihydrogenated graphene 2010, 023107-1-023107-3.

https://ro.uow.edu.au/engpapers/4032

Research Online is the open access institutional repository for the University of Wollongong. For further information contact the UOW Library: research-pubs@uow.edu.au 


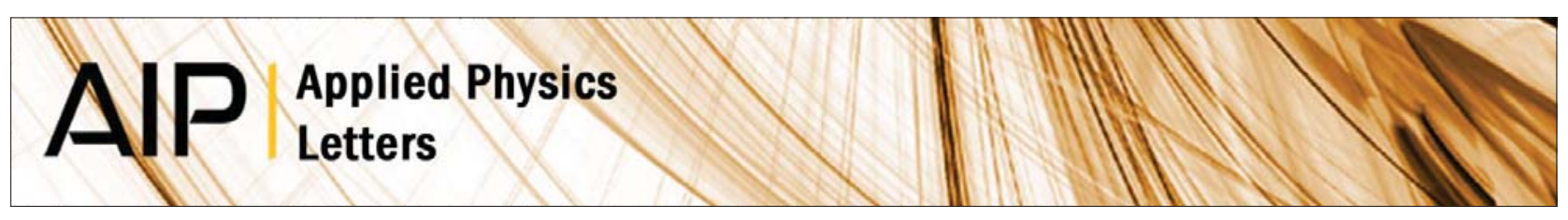

\section{Vertical absorption edge and temperature dependent resistivity in semihydrogenated graphene}

Lei Chen, Zhongshui Ma, and C. Zhang

Citation: Appl. Phys. Lett. 96, 023107 (2010); doi: 10.1063/1.3292026

View online: http://dx.doi.org/10.1063/1.3292026

View Table of Contents: http://apl.aip.org/resource/1/APPLAB/v96/i2

Published by the American Institute of Physics.

Additional information on Appl. Phys. Lett.

Journal Homepage: http://apl.aip.org/

Journal Information: http://apl.aip.org/about/about_the_journal

Top downloads: http://apl.aip.org/features/most_downloaded

Information for Authors: http://apl.aip.org/authors

\section{ADVERTISEMENT}

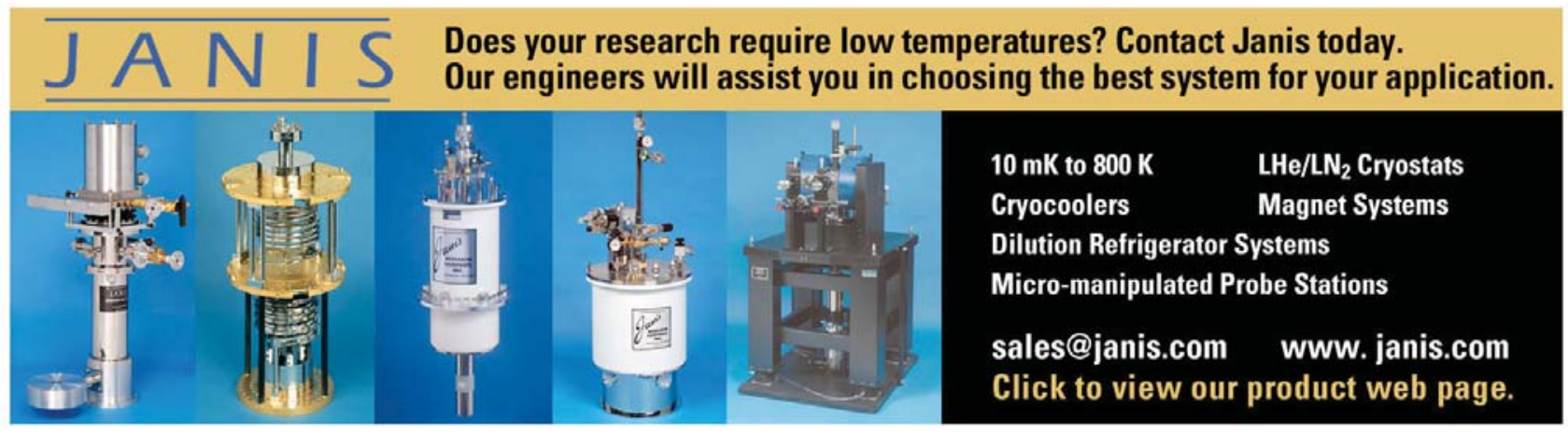




\title{
Vertical absorption edge and temperature dependent resistivity in semihydrogenated graphene
}

\author{
Lei Chen, ${ }^{1, a)}$ Zhongshui Ma, ${ }^{1}$ and C. Zhang ${ }^{2, b)}$ \\ ${ }^{1}$ School of Physics, Peking University, Beijing 100871, People's Republic of China \\ ${ }^{2}$ School of Engineering Physics, University of Wollongong, New South Wales 2552, Australia
}

(Received 28 October 2009; accepted 21 December 2009; published online 12 January 2010)

\begin{abstract}
We show that for graphene with any finite asymmetry in the on-site energy between the two sublattices $(\Delta)$, the optical absorption edge is determined by the $\Delta$. The universal conductance will be broken and the conductance near the band edge varies with frequency as $1 / \omega^{2}$. The onset conductance is $\sigma_{\mathrm{c}}=2 \sigma_{0}=\pi e^{2} / 2 h$, independent of the size of the band gap. The total integrated optical response is nearly conserved despite of the opening of the band gap. Moreover, near the band edge, there is a change over of the electrical resistivity from temperature independent to a linear temperature dependence. (C) 2010 American Institute of Physics. [doi:10.1063/1.3292026]
\end{abstract}

In recent years, graphene has attracted a great deal of interest. ${ }^{1-4}$ Interesting physics have been predicted and observed, such as electron-hole symmetry and half-integer quantum Hall effect, ${ }^{2,3}$ finite conductivity at zero chargecarrier concentration, ${ }^{2}$ and the strong suppression of weak localization. $^{5-7}$ In graphene, the conduction and valence bands touch each other at six equivalent points, the $K$ and $K^{\prime}$ points in the Brillouin zone. Near these points the electrons behave like massless Dirac Fermions. One of the most striking features of the massless Dirac Fermion is that the optical conductance is a universal constant, $\sigma_{0}=\pi e^{2} / 4 h$. This was calculated theoretically long before graphene's fabrication in $2003 .{ }^{8}$ In the visible region of the electromagnetic spectrum, the absorption coefficient and transmittance of graphene have been measured experimentally and the universal conductance has been confirmed. ${ }^{9-11}$

The optical conductivity of graphene outside the low energy Dirac regime has been calculated theoretically. ${ }^{12,13}$ Outside the Dirac regime the band bending results in an increased density of states. As a result the optical response increases and reaches a sharp maximum at the van Hove point of $\epsilon=2 t$ where $\epsilon$ is the electronic energy and $t$ $\approx 3 \mathrm{eV}$ is the hopping bandwidth. The nearly total transparency of graphene can be partially alleviated in the case of bilayer graphene. ${ }^{14}$ The problem is even further alleviated in the case of single layer graphene nanoribbons in a magnetic field where the conductance can be as much as two orders of magnitude higher than that for graphene, ${ }^{15}$ and recently it was shown that a subclass of bilayer nanoribbons is similarly active in the terahertz far-infrared regime even without a magnetic field. ${ }^{16}$

Graphene was predicted and later experimentally confirmed to undergo metal-semiconductor transition when fully hydrogenated (graphane). It is possible that graphene with half of the carbon atoms saturated with hydrogen (semihydrogenated) can become ferromagnetic. ${ }^{17}$ Semihydrogenated graphene loses the symmetry of A and B sublattices. Graphene systems with broken inversion symmetry are of direct experimental interest. Observation of a band gap opening in epitaxial graphene has been reported. ${ }^{18}$ This is a direct

\footnotetext{
${ }^{a)}$ Electronic mail: leichen@pku.edu.cn.

${ }^{b)}$ Electronic mail: czhang@uow.edu.au.
}

consequence of the inversion symmetry breaking by the substrate potential. ${ }^{19}$ A scheme to detect valley polarization in graphene systems with broken inversion symmetry was demonstrated..$^{20}$ In this letter, we shall show that due to the inequivalence of the two valleys, the universal conductance breaks down at low frequencies. If the on-site energy difference of the two sublattice is $\Delta$, absorption is only possible for frequencies above $\Delta$. The conductance at the absorption edge is twice the universal conductance, drops slowly as $1 / \omega^{2}$. Therefore, the universal conductance is only approximately valid in a narrow regime overlapping with the visible band. Furthermore the $\mathrm{AB}$ asymmetry leads to a temperaturedependent resistivity which changes from temperature independent to a linear temperature dependence.

In the tight-binding approximation, the Hamiltonian for the graphene can be written as,

$$
H=\left(\begin{array}{cc}
-t^{\prime} \alpha(\mathbf{k})-\frac{\Delta}{2} & -t H_{12} \\
-t^{*} H_{12}^{*} & -t^{\prime} \alpha(\mathbf{k})+\frac{\Delta}{2}
\end{array}\right),
$$

where $H_{12}=1+e^{i \mathbf{k} \cdot \mathbf{a}_{1}}+e^{i \mathbf{k} \cdot \mathbf{a}_{2}},|t|=2 \hbar v_{F} / \sqrt{3} a$, and $t^{\prime}=\gamma|t|$ is the next nearest neighbor coupling, and $\alpha(\mathbf{k})=2\left[\cos \mathbf{k} \cdot \mathbf{a}_{1}\right.$ $\left.+\cos \mathbf{k} \cdot \mathbf{a}_{2}+\cos \mathbf{k} \cdot\left(\mathbf{a}_{1}-\mathbf{a}_{2}\right)\right] . \mathbf{a}_{1}$ and $\mathbf{a}_{2}$ are unit vectors given as $\mathbf{a}_{1}=a(\sqrt{3} / 2,1 / 2), \mathbf{a}_{2}=a(\sqrt{3} / 2,-1 / 2)$.

In the Hamiltonian, the on-site energies of the A-sublattice and B-sublattice are $-\Delta / 2$ and $\Delta / 2$, respectively. The eigenvalues are

$$
E_{\mathbf{k}, s}=-t^{\prime} \alpha(\mathbf{k})+s \frac{1}{2} \sqrt{\Delta^{2}+4|t|^{2}[3+\alpha(\mathbf{k})]},
$$

with $s= \pm 1$. Correspondingly, the eigenstates can be written in the form of

$$
\psi_{\mathbf{k}, s}(\mathbf{r})=\xi_{\mathbf{k}, s} e^{i \mathbf{k} \cdot \mathbf{r}},
$$

with

$$
\xi_{\mathbf{k}, s}=\frac{1}{\sqrt{2}} \sqrt{1+s \frac{\Delta}{\sqrt{L(\mathbf{k})}}}\left[\begin{array}{c}
-\frac{2 t H_{12}}{\Delta+s \sqrt{L(\mathbf{k})}} \\
1
\end{array}\right],
$$

where $L(\mathbf{k})=\Delta^{2}+4|t|^{2}[3+\alpha(\mathbf{k})]$. The velocity operator can be obtained by $\hat{\mathbf{v}}=(1 / \hbar)(\partial H / \partial \mathbf{k})$ and $\Delta$ is independent of $\mathbf{k}$, 


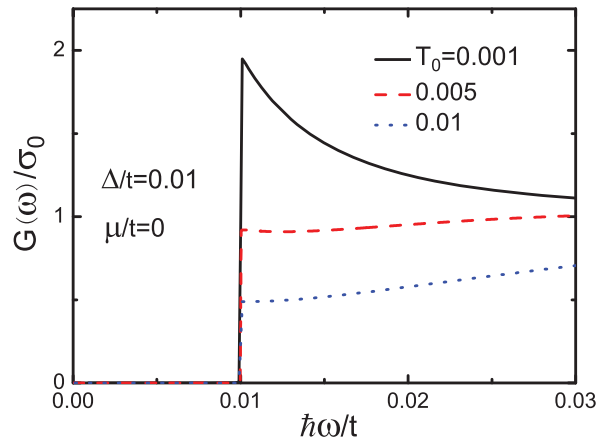

FIG. 1. (Color online) The optical conductance in the low frequency regime, for several different temperatures. Here $T_{0}=k_{\mathrm{B}} T / t$.

$$
\hat{\mathbf{v}}=\frac{1}{\hbar}\left[\begin{array}{cc}
t^{\prime} \mathbf{B}(\mathbf{k}) & -i t C(\mathbf{k}) \\
i t^{*} C^{*}(\mathbf{k}) & t^{\prime} \mathbf{B}(\mathbf{k})
\end{array}\right],
$$

where $\quad \mathbf{B}(\mathbf{k})=\nabla_{\mathbf{k}} \alpha(\mathbf{k})=2\left[\mathbf{a}_{1} \sin \mathbf{k} \cdot \mathbf{a}_{1}+\mathbf{a}_{2} \sin \mathbf{k} \cdot \mathbf{a}_{2}+\left(\mathbf{a}_{1}\right.\right.$ $\left.\left.-\mathbf{a}_{2}\right) \sin \mathbf{k} \cdot\left(\mathbf{a}_{1}-\mathbf{a}_{2}\right)\right]$ and $C(\mathbf{k})=\mathbf{a}_{1} e^{i \mathbf{k} \cdot \mathbf{a}_{1}}+\mathbf{a}_{2} e^{i \mathbf{k} \cdot \mathbf{a}_{2}}$.

The second quantized current density operator is given by

$$
\mathbf{J}=e \sum_{\mathbf{k}, s, s^{\prime}} \xi_{\mathbf{k}, s}^{\dagger} \hat{\mathbf{v}} \xi_{\mathbf{k}, s^{\prime}} a_{\mathbf{k}, s}^{\dagger} a_{\mathbf{k}, s^{\prime}}
$$

The frequency dependent conductivity contains rich information of electronic transitions and plasmonic effect. ${ }^{21}$ The optical conductivity can be calculated from the Kubo formula,

$$
\sigma_{\mu, \nu}(\omega)=\frac{1}{\omega} \int_{0}^{\infty} d t e^{i \omega t}\left\langle\left[J_{\mu}(t), J_{\nu}(0)\right]\right\rangle
$$

In Fig. 1, we plot the optical conductance versus frequency for a typical value of $\Delta=0.03 \mathrm{eV}$ under the nearest neighbor coupling approximation. The A-B on-site energy difference removes the universal conductance which is the key feature of graphene with A-B symmetry. For $\hbar \omega<\Delta$, the conductance is zero for any temperature since only vertical transitions are allowed. For $\hbar \omega>\Delta$, thermal excitation will reduce the carrier concentration near the top of the valence band and the total interband transition rate decreases. As a result, the conductance decreases as temperatures increases. At the frequencies close to the energy gap, the conductance jumps to a maximum which is greater than the universal conductance.

$$
G(\omega)=\sigma_{0}\left[1+\frac{\Delta^{2}}{(\hbar \omega)^{2}}\right] \tanh \left(\frac{\beta}{4} \hbar \omega\right) \Theta(\hbar \omega-\Delta),
$$

where $\sigma_{0}=\pi e^{2} / 4 h$ and $\beta$ is the inverse temperature in energy units. The conductance then decreases slowly as the joint density of states for optical transition decreases.

For systems with complete A-B symmetry, the onset maximum disappears. In this case the conductance starts from the universal conductance at $T=0$ and from zero at finite temperature. The conductance at $\hbar \omega=\Delta$ is $G(\omega)$ $=2 \sigma_{0} \tanh (\beta \Delta / 4)$. In our analysis and conductivity, we have neglected the $t^{\prime}$ term. The effect of $t^{\prime}$ shifts the Fermi level to the valence band and thus suppresses the low frequency conductance. However, one can apply a gate voltage to easily shift the Fermi level to the neutral point.

At relatively low energy around the Dirac points, $\alpha(\mathbf{k})$ $=-3+3 a^{2}(\delta k)^{2} / 4$, and $E_{\mathbf{k},+}-E_{\mathbf{k},-}=\sqrt{L(\mathbf{k})}$, where $\delta k$ is mea-

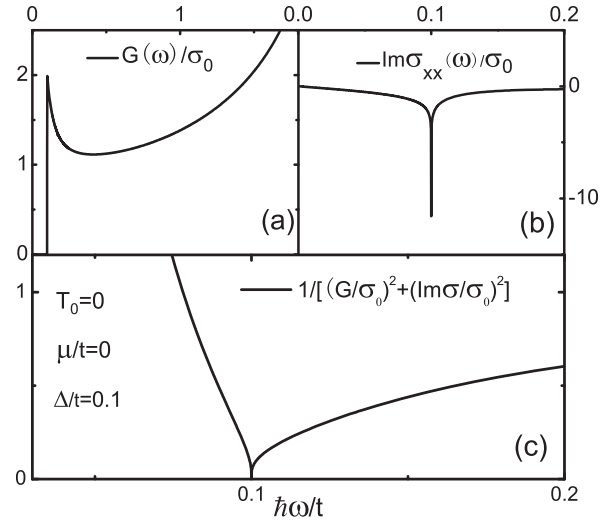

FIG. 2. (a) The optical conductance in the wide frequency regime, (b) the imaginary part of the conductivity (permittivity), and (c) the inverse of $|\sigma(\omega)|^{2}$.

sured from the $K$-point. In this region, the conductance is isotropic, $\operatorname{Re} \sigma_{x x}^{\left(\mathbf{K}_{1}\right)}=\operatorname{Re} \sigma_{y y}^{\left(\mathbf{K}_{1}\right)}=G(\omega)$, given as,

$$
\begin{aligned}
G(\omega)= & -\frac{2 \pi \hbar v_{F}^{2} \sigma_{0}}{\omega} \sum_{\delta \mathbf{k}}\left(\tanh \frac{\beta}{2} E_{\delta \mathbf{k},+}-\tanh \frac{\beta}{2} E_{\delta \mathbf{k},-}\right)\left(1+\frac{\Delta^{2}}{\eta}\right) \\
& \times[\delta(\hbar \omega-\sqrt{\eta})-\delta(\hbar \omega+\sqrt{\eta})] .
\end{aligned}
$$

Here, $\quad \eta(\delta k)=\Delta^{2}+4 \hbar^{2} v_{F}^{2}(\delta k)^{2}$, and $E_{\delta \mathbf{k}, s}=\gamma\left(2 \sqrt{3} \hbar v_{F} / a\right)[1$ $\left.-\frac{\gamma}{4} a^{2}(\delta k)^{2}\right]+s(1 / 2) \sqrt{\eta}$.

In Fig. 2, we show the zero temperature conductance over a wide frequency range of 0 to $2 t$. The absorption at frequencies below the gap $\Delta$ is now forbidden, as expected. The interesting feature of this systems is the onset of absorption at the band edge. The absorption at the edge is discontinuous and jumps vertically from zero to a value twice the universal conductance. This behavior can be derived analytically around the Dirac regime as follows.

In the limit of $T \rightarrow 0, \tan h \frac{1}{2} \beta E_{\mathbf{k},+}-\tan h \frac{1}{2} \beta E_{\mathbf{k},-} \simeq \Theta\left(\Delta^{2}\right.$ $\left.+4 \hbar^{2} v_{F}^{2}(\delta k)^{2}\right)$, we obtain a simple form for the conductance due to the contribution from the six Dirac points, given as $G(\omega)=\sigma_{0}\left[1+\Delta^{2} /(\hbar \omega)^{2}\right] \Theta(\hbar \omega-\Delta)$. At $\hbar \omega=\Delta+0^{+}$for any $\Delta$, the conductance is twice of the universal conductance. In other words, for graphene with A-B asymmetry, while the universal conductance is broken, the onset conductance at the band edge for $T=0$ has a universal value of $2 \sigma_{0}$. In comparison to the well known interband absorption rate in semiconductors, ${ }^{22,23} G_{\text {semicond }}(\omega) \sim \sqrt{\hbar \omega-\Delta}$, this feature of interband absorption in graphene is very unique. We notice that the $2 \sigma_{0}$ onset conductance is consistent with that in the massless Dirac Fermion with an impurity induced gap. ${ }^{9}$ Therefore, this is a very general property of massless Dirac fermion, regardless how the gap is induced. At higher frequency, the conductance increases with frequency, [Fig. 2(a)]. Therefore, an inequivalence of A-B sublattices can remove the universal conductance, or limit its applicability to a small regime around the visible frequency.

The Fig. 2(b) shows the imaginary part of the conductivity. Near the band edge, the imaginary part is very large which indicate phase difference of nearly $\pi / 2$ between the incident field and the current response. This phase difference decreases rapidly as frequency increases. The size of the dip at $\hbar \omega=\Delta$ is about the same order of magnitude as the jump in the conductance. Figure 2(c) shows the inverse of the absolute value of the conductivity squared. This quantity is directly related to the transmittance near the band gap. Due 


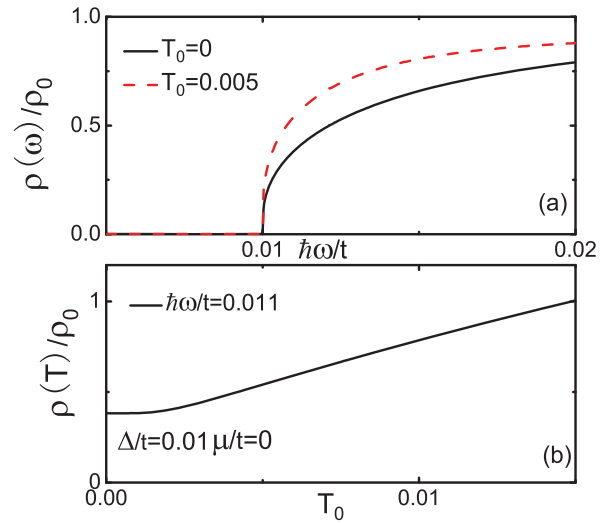

FIG. 3. (Color online) (a) The frequency dependent resistivity near the band edge. (b) The temperature dependence of the resistivity at low frequencies.

to the large permittivity near the gap, the transmittance is significantly low than that of $97 \%$ in the visible range for graphene.

The total integrated absorption is given by

$$
\Lambda_{\text {total }}=\int d \omega G(\omega)
$$

Experimentally, ${ }^{11}$ it has been shown that for graphene with A-B symmetry, the universal conductance is valid up to the high end of the visible band, $\lambda=400 \mathrm{~nm}$ or $\hbar \omega=2 \mathrm{eV}$. Theoretically it is shown that the conductance is approximately $\sigma_{0}$ up to $\hbar \omega=t$. Therefore for graphene with A-B symmetry, we integrate (8) from 0 to $t$ and $\sigma_{\text {total }}=2 t \sigma_{0}$. For graphene without A-B symmetry, we integrate (8) from $\Delta$ to $t$ and $\sigma_{\text {total }}=2 t \sigma_{0}\left(1-\Delta^{2} / t^{2}\right)$. Since $\Delta^{2} / t^{2} \approx 0.01$, we can conclude that the total optical response is nearly conserved even when a gap opens up at the Fermi energy. For small $\Delta$, any loss of optical response due to the gap is recovered in the regime of $\hbar \omega$ slightly higher than $\Delta$. For large $\Delta$, the region required for recovering the optical response is wider.

The quantity that determines the transport characteristics of graphene is the resistivity, defined as $\rho(\omega)=\operatorname{Re}[1 / \sigma(\omega)]$. In Fig. 3(a), we show the frequency dependence of the resistivity at two different temperatures. Comparing to the conductance, the resistivity also starts vertically at the band edge. However its curvature is finite and it is monotonic function of frequency. For frequency much higher than the band gap, the saturation value of the resistivity is $1 / \sigma_{0}$. The most interesting feature of the resistivity is its temperature dependence, shown in Fig. 3(b). There exists a characteristic temperature, $T_{\mathrm{c}}$, separating the two distinctive temperature regime. At $\hbar \omega=0.011 t, T_{\mathrm{c}} \approx 0.002 t \approx 70 \mathrm{~K}$. For our system of disorder-free graphene, the resistivity is solely due to the interband transitions. Below $T_{\mathrm{c}}$, the carrier thermalisation is negligible and the resistivity is temperature independent. Further increasing $T$ to above $T_{\mathrm{c}}$, the thermal population of carriers in both bands reduces the phase space for photoresponse and increases the thermalisation. The linear temperature dependence of resistivity has been the feature of layer high temperature superconductors where scattering plays an important role. The mechanism of line- $T$ dependence in the present system is different. It is due to the reduced photoelectrons by thermalisation. The change over of the resistivity characteristics is controlled by the interplay of thermal energy, band gap, and the frequency.

In conclusion, we have shown that for graphene without inversion symmetry the optical conductance becomes strongly frequency dependent. There exists a characteristic temperature at which the resistivity from being temperature independent to a linear temperature dependence.

This work is supported in part by the National Natural Science Foundation of China (Grant Nos. 10674004 and 10874002) and the Australian Research Council.

${ }^{1}$ K. S. Novoselov, A. K. Geim, S. V. Morozov, D. Jiang, Y. Zhang, S. V. Dubonos, I. V. Grigorieva, and A. A. Firsov, Science 306, 666 (2004).

${ }^{2}$ K. S. Novoselov, A. K. Geim, S. V. Morozov, D. Jiang, M. I. Katsenelson, I. V. Grigorieva, S. V. Dubonos, and A. A. Firsov, Nature (London) 438, 197 (2005).

${ }^{3}$ Y. Zhang, Y. W. Tan, H. L. Stormer, and P. Kim, Nature (London) 438, 201 (2005)

${ }^{4}$ C. Berger, Z. Song, X. Li, X. Wu, N. Brown, C. Naud, D. Mayou, T. Li, J. Hass, A. N. Marchenkov, E. H. Konrad, P. N. First, and W. A. de Heer, Science 312, 1191 (2006).

${ }^{5}$ H. Suzuura and T. Ando, Phys. Rev. Lett. 89, 266603 (2002).

${ }^{6}$ S. V. Morozov, K. S. Novoselov, M. I. Katsnelson, F. Schedin, L. A. Ponomarenko, D. Jiang, and A. K. Geim, Phys. Rev. Lett. 97, 016801 (2006).

${ }^{7}$ D. V. Khveshchenko, Phys. Rev. Lett. 97, 036802 (2006).

${ }^{8}$ E. Fradkin, Phys. Rev. B 33, 3263 (1986).

${ }^{9}$ V. P. Gusynin, S. G. Shrapov, and J. P. Carbotte, Phys. Rev. Lett. 96, 256802 (2006).

${ }^{10}$ A. B. Kuzmenko, E. van Heumen, F. Carbone, and D. van der Marel, Phys. Rev. Lett. 100, 117401 (2008).

${ }^{11}$ R. R. Nair, P. Blake, A. N. Grigorenko, K. S. Novoselov, T. J. Booth, T. Stauber, N. M. R. Peres, and A. K. Geim, Science 320, 1308 (2008).

${ }^{12}$ C. Zhang, L. Chen, and Z. Ma, Phys. Rev. B 77, 241402 (2008).

${ }^{13}$ T. Stauber, N. M. R. Peres, and A. K. Geim, Phys. Rev. B 78, 085432 (2008).

${ }^{14}$ A. R. Wright, F. Liu, and C. Zhang, Nanotechnology 20, 405203 (2009).

${ }^{15}$ J. Liu, A. R. Wright, C. Zhang, and Z. Ma, Appl. Phys. Lett. 93, 041106 (2008).

${ }^{16}$ A.R. Wright, J.C. Cao, C.Zhang, Phys. Rev. Lett. 103, 207401 (2009).

${ }^{17}$ J. Zhou, Q. Wang, Q. Sun, X. S. Chen, Y. Kawazoe, and P. Jena, Nano Lett. 9, 3867 (2009).

${ }^{18}$ S. Y. Zhou, G.-H. Gweon, A. V. Fedorov, P. N. First, W. A. de Heer, D.-H. Lee, F. Guinea, A. H. Castro Neto, and A. Lanzara, Nature Mater. 6, 770 (2007).

${ }^{19}$ S. Gwo and C. K. Shih, Phys. Rev. B 47, 13059 (1993).

${ }^{20}$ D. Xiao, W. Yao, and Q. Niu, Phys. Rev. Lett. 99, 236809 (2007).

${ }^{21}$ C. Zhang, Phys. Rev. B 66, 081105 (2002).

${ }^{22}$ B. Ullrich, C. Zhang, and K. von Klitzing, Appl. Phys. Lett. 54, 1133 (1989).

${ }^{23}$ K. Seeger, Semiconductor Physics, 3rd ed. (Springer, New York, 1986), p. 340 . 